\title{
EXPOSED FACES OF THE UNIT BALL IN A $\mathrm{JBW}^{*}$-TRIPLE ${ }^{1}$
}

\author{
C. MARTIN EDWARDS and GOTTFRIED T. RÜTTIMANN
}

\begin{abstract}
.
A tripotent in a $\mathrm{JBW}^{*}$-triple $A$ is said to be $\sigma$-finite if it does not majorize an uncountable orthogonal subset of tripotents in $A$. It is shown that every $\mathrm{JBW}^{*}$-triple is weak*-linearly generated by its set of $\sigma$-finite tripotents.

A JBW*-triple $A$ is said to be $\sigma$-finite if every tripotent in $A$ is $\sigma$-finite. Characterizations of $\sigma$ finiteness of a $\mathrm{JBW}^{*}$-triple are given in terms of the geometry of the unit ball $A_{1}$ and in terms of lattice-theoretic properties of the partially ordered set of its tripotents. It is shown that $\sigma$-finiteness of $A$ is equivalent to each of the properties that every weak*-closed face of $A_{1}$ is weak*exposed, that $A_{1}$ has a weak*-exposed point, that $A_{1}$ has a norm-exposed point, and that $A$ has a $\sigma$-finite maximal tripotent. These results are applied to $\mathrm{W}^{*}$-algebras, to certain sub-JBW*-triples of $\mathrm{W}^{*}$-algebras and to spin triples.
\end{abstract}

\section{Introduction.}

This paper presents a further investigation into the facial structure of the unit balls in a Banach space and its dual. In previous papers the authors investigated the facial structure and identified certain classes of faces of the unit balls in GL-spaces and their duals, in JBW-algebras and their preduals, in GM-spaces and their duals and in JB-algebras and their duals [8], [9]. Many of the techniques developed and used in those papers do not easily extend to the case where the Banach spaces in question are taken over the complex field. Using different methods, in [10], the authors completed a study of the facial structure of the unit ball in a $\mathrm{JBW}^{*}$-triple $A$ and its predual $A_{*}$. It was shown that every norm-closed face of the unit ball $A_{*, 1}$ of $A_{*}$ is norm-exposed and that every weak*-closed face of the unit ball $A_{1}$ of $A$ is weak*-semi-exposed. Moreover, the facial geometry of the unit balls $A_{1}$ and $A_{*, 1}$ is closely related to the algebraic structure of $A$. In fact, the complete lattice of norm-closed faces of $A_{*, 1}$ is order isomorphic to the complete lattice $\mathscr{U}(A)$ consisting of the partially ordered set $\mathscr{U}(A)$ of tripotents in $A$ with

\footnotetext{
1 Research partially supported by Schweizerischer Nationalfonds/Fonds national suisse.

Received December 12, 1995.
} 
a greatest element adjoined, and the complete lattice of weak*-closed faces of $A_{1}$ is anti-order isomorphic to $\mathscr{U}(A)$.

This paper casts some light upon the identification of those $\mathrm{JBW}^{*}$-triples which have the property that every weak*-closed face of their unit ball is weak*-exposed. A tripotent in a $\mathrm{JBW}^{*}$-triple $A$ is said to be $\sigma$-finite if it does not majorize an uncountable orthogonal subset of $\mathscr{U}(A)$. It is a remarkable fact that, not only do non-zero $\sigma$-finite tripotents exist in every non-trivial $\mathrm{JBW}^{*}$-triple, but also the set $\mathscr{U}_{\sigma}(A)$ of $\sigma$-finite tripotents in $A$ weak*-linearly generates $A$. A $\mathrm{JBW}^{*}$-triple is said to be $\sigma$-finite if every tripotent in $A$ is $\sigma$ finite. It is shown that a $\mathrm{JBW}^{*}$-triple $A$ has the property that every weak*closed face of its unit ball $A_{1}$ is weak*-exposed if and only if it is $\sigma$-finite. Somewhat surprisingly, it follows that either of these conditions is equivalent to the condition that $A$ contains a single maximal $\sigma$-finite tripotent, to the condition that $A_{1}$ contains a single norm-exposed point, and to the condition that $A_{1}$ contains a single weak*-exposed point.

This paper is organized as follows. In $\$ 2$ definitions are given, notation is established, and certain preliminary results are proved. In $\S 3$ the properties of the set of $\sigma$-finite tripotents in an arbitrary $\mathrm{JBW}^{*}$-triple are studied and in $\S 4$ the main results are proved. In $\S 5$ the results of $\S 4$ are applied to $W^{*}$-algebras, certain sub-JBW*-triples of a $\mathrm{W}^{*}$-algebra and to other examples of $\mathrm{JBW}^{*}$-triples. In particular, it is shown that a $\mathrm{W}^{*}$-algebra is $\sigma$-finite as a $\mathrm{JBW}^{*}$-triple if and only if it is $\sigma$-finite as a $\mathrm{W}^{*}$-algebra, thereby leading to several new characterizations of $\sigma$-finite $\mathrm{W}^{*}$-algebras.

\section{Preliminaries.}

A Jordan *-algebra $A$ which is also a complex Banach space such that, for all elements $a$ and $b$ in $A,\left\|a^{*}\right\|=\|a\|,\|a \circ b\| \leq\|a\|\|b\|$ and $\left\|\left\{\begin{array}{lll}a & a & a\end{array}\right\}\right\|=\|a\|^{3}$, where

$$
\{a b c\}=a \circ\left(b^{*} \circ c\right)+\left(a \circ b^{*}\right) \circ c-b^{*} \circ(a \circ c)
$$

is the Jordan triple product on $A$, is said to be a Jordan $C^{*}$-algebra [30] or $J B^{*}$-algebra [31]. A Jordan $\mathrm{C}^{*}$-algebra which is the dual of a Banach space is said to be a Jordan $W^{*}$-algebra [6] or a $J B W^{*}$-algebra [31]. Examples of $\mathrm{JB}^{*}$ algebras are $\mathrm{C}^{*}$-algebras and examples of $\mathrm{JBW}^{*}$-algebras are $\mathrm{W}^{*}$-algebras in both cases equipped with the Jordan product

$$
a \circ b=\frac{1}{2}(a b+b a) .
$$

The self-adjoint part $A_{\mathrm{sa}}$ of a $\mathrm{JB}^{*}$-algebra $A$ is a JB-algebra and the self-adjoint part of a $\mathrm{JBW}^{*}$-algebra is a $\mathrm{JBW}$-algebra [22]. For the properties of $\mathrm{C}^{*}$ - 
algebras and $\mathrm{W}^{*}$-algebras the reader is referred to [27], [28] and for the algebraic properties of Jordan algebras to [22], [24], [25], [26].

A complex vector space $A$ equipped with a triple product $(a, b, c) \mapsto\{a b c\}$ from $A \times A \times A$ to $A$ which is symmetric and linear in the first and third variables, conjugate linear in the second variable and satisfies the identity

$$
[D(a, b), D(c, d)]=D(\{a b c\}, d)-D(c,\{d a b\})
$$

where [,] denotes the commutator and $D$ is the mapping from $A \times A$ to $A$ defined by

$$
D(a, b) c=\{a b c\}
$$

is said to be a Jordan*-triple. A subspace $B$ of a Jordan*-triple $A$ is said to be a subtriple if $\{B B B\}$ is contained in $B$ and is said to be an inner ideal if $\{B A B\}$ is contained in $B$. When $A$ is also a Banach space such that $D$ is continuous from $A \times A$ to the Banach space $B(A)$ of bounded linear operators on $A$, and, for each element $a$ in $A, D(a, a)$ is hermitian with non-negative spectrum and satisfies

$$
\|D(a, a)\|=\|a\|^{2},
$$

then $A$ is said to be a $\mathrm{JB}^{*}$-triple. A $\mathrm{JB}^{*}$-triple which is the dual of a Banach space is called a $\mathrm{JBW}^{*}$-triple. Examples of $\mathrm{JB}^{*}$-triples are $\mathrm{JB}^{*}$-algebras and examples of $\mathrm{JBW}^{*}$-triples are $\mathrm{JBW}^{*}$-algebras both with respect to the triple product above. The second dual $A^{* *}$ of a $\mathrm{JB}^{*}$-triple $A$ is a $\mathrm{JBW}^{*}$-triple.

An element $u$ in a $\mathrm{JBW}^{*}$-triple $A$ is said to be a tripotent if $\{u u u\}$ is equal to $u$. The set of tripotents in $A$ is denoted by $\mathscr{U}(A)$. Notice that the weak*closure of the linear hull of $\mathscr{U}(A)$ coincides with $A$. For each tripotent $u$ in the $\mathrm{JBW}^{*}$-triple $A$, the weak* ${ }^{*}$-continuous conjugate linear operator $Q(u)$ and the weak ${ }^{*}$-continuous linear operators $P_{j}(u), j=0,1,2$, are defined, for each element $a$ in $A$, by

$$
\begin{aligned}
Q(u) a=\{u a u\}, & P_{2}(u)=Q(u)^{2}, \\
P_{1}(u)=2\left(D(u, u)-Q(u)^{2}\right), & P_{0}(u)=I-2 D(u, u)+Q(u)^{2} .
\end{aligned}
$$

The linear operators $P_{j}(u), j=0,1,2$, are projections onto the eigenspaces $A_{j}(u)$ of $D(u, u)$ corresponding to eigenvalues $j / 2$ and

$$
A=A_{0}(u) \oplus A_{1}(u) \oplus A_{2}(u)
$$

is the Peirce decomposition of $A$ relative to $u$. For $i, j, k=0,1,2, A_{i}(u)$ is a weak*-closed subtriple of $A$ such that $\left\{A_{i}(u) A_{j}(u) A_{k}(u)\right\} \subseteq A_{i-j+k}(u)$ when $i-j+k=0,1$, or 2 , and $\{0\}$ otherwise, and

$$
\left\{A_{2}(u) A_{0}(u) A\right\}=\left\{A_{0}(u) A_{2}(u) A\right\}=\{0\} .
$$


Notice that $A_{0}(u)$ and $A_{2}(u)$ are inner ideals in $A$. With respect to the separately weak ${ }^{*}$-continuous product $(a, b) \mapsto a \circ b=\{a u b\}$ and the norm-preserving involution $a \mapsto a^{\dagger}=\{u a u\}, A_{2}(u)$ is a $\mathrm{JBW}^{*}$-algebra with unit $u$. For details the reader is referred to [1], [3], [4], [5], [7], [10], [12], [13], [14], [15], [16], [17], [19], [20], [23].

A pair $u, v$ of elements in $\mathscr{U}(A)$ is said to be orthogonal, denoted by $u \perp v$, if $v$ is contained in $A_{0}(u)$. It can be seen that $\perp$ is a symmetric relation on $\mathscr{U}(A)$. A subset $M$ in $\mathscr{U}(A)$ is said to be orthogonal if, for every pair $u, v$ of tripotents in $M$ with $u \neq v, u \perp v$. For two elements $u$ and $v$ in $\mathscr{U}(A)$, write $u \leq v$ if $\{u v u\}=u$ or, equivalently, if $v-u$ is a tripotent orthogonal to $u$.

Let $A$ be a $\mathrm{JBW}^{*}$-algebra and let $\mathscr{P}(A)$ be the collection of the self-adjoint idempotents, the projections, in $A$. Clearly, $\mathscr{P}(A)$ is a subset of $\mathscr{U}(A)$. Moreover, for elements $p, q$ in $\mathscr{P}(A), p \leq q$ if and only if $p \circ q=p$ and $p \perp q$ if and only if $p \circ q=0$.

Basic properties of the two binary relations introduced above are summarized below. In the sequel we shall make frequent use of these.

Theorem 2.1. Let $A$ be a $\mathrm{JBW}^{*}$-triple, let $\mathscr{U}(A)$ be the collection of tripotents in $A$ and let $\perp$ and $\leq$ be the binary relations defined above. Then:

(i) The relation $\leq$ is a partial ordering on $\mathscr{U}(A)$ and 0 is the least element in $\mathscr{U}(A)$.

(ii) For an element $u$ in $\mathscr{U}(A), u \perp u$ if and only if $u$ is equal to 0 .

(iii) If $u$ and $v$ are elements in $\mathscr{U}(A)$ such that $u \perp v$, then $u \vee v$ exists and is equal to $u+v$.

(iv) If $u$ and $v$ are elements in $\mathscr{U}(A)$ such that $u \leq v$, then there exists a unique element $w$ in $\mathscr{U}(A)$ such that $w \perp u$ and $u \vee w$ is equal to $v$.

v) If $u, v$ and $w$ are elements in $\mathscr{U}(A)$ such that $u \leq v$ and $v \perp w$ then $u \perp w$.

(vi) Let $\left(u_{\gamma}\right)_{\gamma \in \Gamma}$ be a family of elements in $\mathscr{U}(A)$. Then $\bigwedge_{\gamma \in \Gamma} u_{\gamma}$ exists.

(vii) Let $\left(u_{\gamma}\right)_{\gamma \in \Gamma}$ be an increasing net in $\mathscr{U}(A)$. Then $\bigvee_{\gamma \in \Gamma} u_{\gamma}$ exists.

(viii) Let $\left(u_{\gamma}\right)_{\gamma \in \Gamma}$ be a family of elements of $\mathscr{U}(A)$ such that $\bigvee_{\gamma \in \Gamma} u_{\gamma}$ exists and let $u$ be an element of $\mathscr{U}(A)$. If, for all $\gamma$ in $\Gamma, u_{\gamma} \perp u$ then $\bigvee_{\gamma \in \Gamma} u_{\gamma} \perp u$.

Proof. See [4] and [23].

It is easily seen that $\mathscr{U}(A)$ has a greatest element if and only if $A$ coincides with $\{0\}$. It is an immediate consequence of Theorem 1 that the partially ordered set $\mathscr{U}(A)$ contains maximal elements and that every tripotent in $A$ is majorized by a maximal element.

Corollary 2.2. Let $A$ be a $\mathrm{JBW}^{*}$-triple, let $u$ be a tripotent in $A$ and let $[0, u]$ be the order interval $\{v \in \mathscr{U}(A): 0 \leq v \leq u\}$. Then, with respect to the restricted ordering, $[0, u]$ forms a complete lattice with least element 0 and greatest element $u$. The supremum and infimum of any non-empty subset of 
$[0, u]$ act as supremum and infimum, respectively, in the partially ordered set $\mathscr{U}(A)$.

Proof. This follows directly from Theorem 2.1 .

TheOREm 2.3. Let $A$ be a $\mathrm{JBW}^{*}$-triple and let $\mathscr{U}(A)$ be the partially ordered set of tripotents in $A$. Let $M$ be an orthogonal subset of $\mathscr{U}(A)$ and let $M^{f}$ be the collection of finite subsets of $M$ upward directed by set-inclusion. Then the supremum $\bigvee_{u \in M} u$ of $M$ exists in $\mathscr{U}(A)$ and is equal to the weak*-limit of the weak*-convergent increasing net $\left(\bigvee_{u \in N} u\right)_{N \in M^{f}}$.

Proof. See [4] or [23].

Let $\mathscr{U}(A)^{\sim}$ be the union of the set $\mathscr{U}(A)$ and the one point set $\left\{u_{\infty}\right\}$ and extend the partial ordering $\leq$ to $\mathscr{U}(A)^{\sim}$ by defining $u \leq u_{\infty}$ for all elements $u$ in $\mathscr{U}(A)^{\sim}$. Then, by Theorem 2.1, $\mathscr{U}(A)^{\sim}$ is a complete lattice referred to as the lattice of tripotents in $A$.

Let $V$ be a complex vector space and let $C$ be a convex subset of $V$. A convex subset $E$ of $C$ is said to be a face of $C$ provided that, if $t x_{1}+(1-t) x_{2}$ is an element of $E$, where $x_{1}$ and $x_{2}$ lie in $C$ and $0<t<1$, then $x_{1}$ and $x_{2}$ lie in $E$. An element $x$ in $C$ is called an extreme point of $C$ if the subset $\{x\}$ is a face of $C$. Let $\tau$ be a locally convex Hausdorff topology on $V$ and let $C$ be $\tau$-closed. Let $\mathscr{F}_{\tau}(C)$ denote the set of all $\tau$-closed faces of $C$. Both $\emptyset$ and $C$ are elements of $\mathscr{F}_{\tau}(C)$ and the intersection of an arbitrary family of elements of $\mathscr{F}_{\tau}(C)$ again lies in $\mathscr{F}_{\tau}(C)$. Hence, with respect to the ordering by set inclusion, $\mathscr{F}_{\tau}(C)$ forms a complete lattice. A subset $E$ of $C$ is said to be a $\tau$-exposed face of $C$ if there exists a $\tau$-continuous linear functional $f$ on $V$ and a real number $t$ such that, for all elements $x$ in $C \backslash E$, $\operatorname{Re} f(x)$ is less than $t$ and, for all elements $x$ in $E, \operatorname{Re} f(x)$ is equal to $t$. An element $x$ is called a $\tau$-exposed point of $C$ if the subset $\{x\}$ is a $\tau$-exposed face of $C$. Let $\mathscr{E}_{\tau}(C)$ denote the set of $\tau$-exposed faces of $C$. Clearly, $\mathscr{E}_{\tau}(C)$ is contained in $\mathscr{F}_{\tau}(C)$ and the intersection of a finite number of elements of $\mathscr{E}_{\tau}(C)$ again lies in $\mathscr{E}_{\tau}(C)$. Moreover, both $\emptyset$ and $C$ belong to $\mathscr{E}_{\tau}(C)$. Also notice that a $\tau$-exposed point of $C$ is an extreme point of $C$. The intersection of an arbitrary family of elements of $\mathscr{E}_{\tau}(C)$ is said to be a $\tau$-semi-exposed face of $C$. Let $\mathscr{S}_{\tau}(C)$ denote the set of $\tau$-semi-exposed faces of $C$. Clearly $\mathscr{E}_{\tau}(C)$ is contained in $\mathscr{S}_{\tau}(C)$ and the intersection of an arbitrary family of elements of $\mathscr{S}_{\tau}(C)$ again lies in $\mathscr{S}_{\tau}(C)$. Hence, with respect to the ordering by set inclusion $\mathscr{S}_{\tau}(C)$ forms a complete lattice and the infimum of a family of elements of $\mathscr{S}_{\tau}(C)$ coincides with its infimum when taken in $\mathscr{F}_{\tau}(C)$.

When $V$ is a complex Banach space with dual space $V^{*}$ the abbreviations $n$ and $w^{*}$ will be used for the norm topology of $V$ and the weak* topology of $V^{*}$, respectively. For each subset $E$ of the unit ball $V_{1}$ in $V$ and $F$ of the unit 
ball $V_{1}^{*}$ of $V^{*}$ let the subsets $E^{\prime}$ and $F$, be defined by

$$
E^{\prime}=\left\{a \in V_{1}^{*}: a(x)=1 \forall x \in E\right\}, \quad F_{\prime}=\left\{x \in V_{1}: a(x)=1 \forall a \in F\right\} .
$$

Notice that $E$ lies in $\mathscr{S}_{n}\left(V_{1}\right)$ if and only if $\left(E^{\prime}\right)$, coincides with $E, F$ lies in $\mathscr{S}_{w^{*}}\left(V_{1}^{*}\right)$ if and only if $\left(F_{l}\right)^{\prime}$ coincides with $F$ and the mappings $E \mapsto E^{\prime}$ and $F \mapsto F$, are anti-order isomorphisms between the complete lattices $\mathscr{S}_{n}\left(V_{1}\right)$ and $\mathscr{S}_{w^{*}}\left(V_{1}^{*}\right)$ and are inverses of each other. The reader is referred to [8], [9], [10] for details.

Let $A$ be a $\mathrm{JBW}^{*}$-triple with predual Banach space $A_{*}$. The tripotents in $A$ are contained in the unit ball $A_{1}$ of $A$. Given a tripotent $u$ in $A$ then, by the above, $\{u\}$, is a norm-exposed face of the unit ball $A_{*, 1}$ of $A_{*}$ and $\{u\}_{\text {, }}^{\prime}$ is a weak*-semi-exposed face of the the unit ball $A_{1}$ of $A$. Notice that, for each element $u$ in $\mathscr{U}(A)$, the convex subsets $\{u\}, \subseteq A_{*, 1}$ and $\{u\}^{\prime} \subseteq A^{* *}{ }_{1}$ are affinely equivalent to the normal state space and the state space, respectively, of the $\mathrm{JBW}^{*}$-algebra $A_{2}(u)$. We define $\left\{u_{\infty}\right\}$, to be the set $A_{*, 1}$.

The following results play an important role in the course of these investigations.

Theorem 2.4. Let $A$ be a $\mathrm{JBW}^{*}$-triple with predual Banach space $A_{*}$. Then the mapping $u \mapsto\{u\}$, is an order isomorphism from the lattice of tripotents $\mathscr{U}(A)^{\sim}$ in $A$ onto the complete lattice $\mathscr{F}_{n}\left(A_{*, 1}\right)$ of norm-closed faces of the unit ball $A_{*, 1}$ of $A_{*}$.

Proof. See [10], Theorem 4.4.

Theorem 2.5. Let $A$ be a $\mathrm{JBW}^{*}$-triple with predual Banach space $A_{*}$. Then the mapping $u \mapsto\{u\}$,' is an anti-order-isomorphism from $\mathscr{U}(A)^{-}$onto the complete lattice $\mathscr{F}_{w^{*}}\left(A_{1}\right)$ of weak*-closed faces of the unit ball $A_{1}$ of $A$. Moreover, for every element $u$ in $\mathscr{U}(A)$,

$$
\{u\}_{\prime}^{\prime}=u+A_{0}(u) \cap A_{1} .
$$

Proof. See [10], Theorem 4.6.

Corollary 2.6. Let $A$ be a $\mathrm{JBW}^{*}$-triple and let $u$ be a tripotent in $A$. Then the subset $\{u\},{ }^{\prime}$ is the smallest weak* ${ }^{*}$ closed face of $A_{1}$ containing $u$.

Proof. Let $F$ be a weak*-closed face containing $u$. By Theorem 2.5 , there exists an element $v$ in $\mathscr{U}(A)$ such that $F$ coincides with $\{v\}_{\prime}^{\prime}$. Then, clearly,

$$
\{u\}_{,}^{\prime} \subseteq\{v\}_{,}^{\prime},^{\prime}=F .
$$

Let $A$ be a $\mathrm{JBW}^{*}$-triple with predual Banach space $A_{*}$. By [19], Proposition 2, for every element $x$ in $A_{*}$, there exists a unique tripotent $e(x)$ in $A$ such that $x$ lies in $A_{*, 2}(e(x))$ and the restriction of $x$ to $A_{2}(e(x))$ is a faithful 
normal positive linear functional on the $\mathrm{JBW}^{*}$-algebra $A_{2}(e(x))$. The tripotent $e(x)$ is called the support of $x$. It has been shown in [10], Lemma 3.6, that the support $e(x)$ of an element $x$ in $A_{*}$ is the least tripotent in $A$ such that $x(e(x))$ is equal to $\|x\|$. If $x$ is of norm one then the face $\{x\}^{\prime}$, of $A_{1}$ coincides with the face $\{e(x)\}$.

\section{The Set of $\sigma$-Finite Tripotents.}

Let $A$ be a $\mathrm{JBW}^{*}$-triple and let $\mathscr{U}(A)$ be the collection of tripotents in $A$. A tripotent $u$ in $A$ is said to be $\sigma$-finite if $u$ does not majorize an uncountable orthogonal subset of tripotents in $A$. The collection of $\sigma$-finite tripotents in $A$ is denoted by $\mathscr{U}_{\sigma}(A)$. Clearly, 0 lies in $\mathscr{U}_{\sigma}(A)$. If $u$ is an element in $\mathscr{U}_{\sigma}(A)$ then the order interval $[0, u]$ is contained in $\mathscr{U}_{\sigma}(A)$.

A $\mathrm{JBW}^{*}$-triple $A$ is said to be $\sigma$-finite if every tripotent $u$ in $A$ is $\sigma$-finite. Clearly, a weak ${ }^{*}$-closed subtriple of a $\sigma$-finite $\mathrm{JBW}^{*}$-triple is a $\sigma$-finite $\mathrm{JBW}^{*}$ triple in its own right.

Proposition 3.1. Let $A$ be a $\mathrm{JBW}^{*}$-triple. Then $A$ is $\sigma$-finite if and only if every orthogonal subset of tripotents in $A$ is at most countable.

Proof. Let $A$ be $\sigma$-finite and let $M$ be an orthogonal subset of tripotents. By Theorem 2.3, the supremum of $M$ exists in $\mathscr{U}(A)$ which is clearly an upper bound of $M$. Therefore the subset $M$ is at most countable. The converse is immediate.

Theorem 3.2. Let $A$ be a $\mathrm{JBW}^{*}$-triple with predual Banach space $A_{*}$. A tripotent $u$ in $A$ is $\sigma$-finite if and only if there exists an element $x$ in $A_{*}$ the support $e(x)$ of which coincides with $u$.

Proof. Let $u$ be a non-zero element in $\mathscr{U}_{\sigma}(A)$. Then every orthogonal subset of self-adjoint idempotents in the $\mathrm{JBW}^{*}$-algebra $A_{2}(u)$ is at most countable. By [8], Theorem 4.6, $A_{2}(u)$ then admits a faithful normal state $x$. Since $A_{2}(u)$ is a weak ${ }^{*}$-closed inner ideal in $A$, by [12], Theorem 2.5, the functional $x$ has a unique extension to a weak ${ }^{*}$-continuous linear functional $\tilde{x}$ of norm one on $A$. Since $\tilde{x}(u)$ is equal to 1 it follows that $e(\tilde{x}) \leq u$ and therefore, by [10], Lemma $2.4, e(\tilde{x})$ is a self-adjoint idempotent in $A_{2}(u)$. Since

$$
x(e(\tilde{x}))=\tilde{x}(e(\tilde{x}))=1
$$

and $x$ is faithful on $A_{2}(u)$ we conclude that $e(\tilde{x})$ is equal to $u$.

Conversely, let $x$ be an element in $A_{*}$ of norm one. By definition, $\left.x\right|_{A_{2}(e(x))_{\text {sa }}}$ is a faithful normal state on the JBW-algebra $A_{2}(e(x))_{s a}$. Let $M$ be a nonempty orthogonal subset of tripotents majorized by $e(x)$. Then the elements 
of $M$ are idempotents in $A_{2}(e(x))_{\text {sa }}$ and therefore, by [8], Theorem 4.6, the set $M$ is at most countable.

Corollary 3.3. Let $A$ be a $\mathrm{JBW}^{*}$-triple and let $A_{1}$ be its unit ball. Let $u$ be a tripotent in $A$. Then the following conditions are equivalent.

(i) The tripotent $u$ is $\sigma$-finite.

(ii) Every weak*-closed face containing $u$ is weak*-exposed.

(iii) The weak*-closed face of $A_{1}$ generated by $u$ is weak ${ }^{*}$-exposed.

Proof. (i) $\Rightarrow$ (ii): Let $F$ be a weak*-closed face of $A_{1}$ containing $u$. By Theorem 2.5, there exists a tripotent $v$ such that $\{v\}{ }^{\prime}$ coincides with $F$. It follows that $v \leq u$ and, consequently, that $v$ is $\sigma$-finite. By Theorem 3.2, there exists an element $x$ in $A_{*, 1}$ such that $v$ is equal to the support $e(x)$ of $x$. Then

$$
F=\{v\}_{,}^{\prime}=\{e(x)\}_{,}^{\prime}=\{x\}^{\prime}
$$

and therefore the face $F$ is weak*-exposed.

(ii) $\Rightarrow$ (iii): This is straightforward.

(iii) $\Rightarrow$ (i): If $u$ is different from zero then $\{u\}_{,}^{\prime}$ differs from $A_{1}$. By Corollary 2.6 and the hypothesis, there exists an element $x$ of norm one in $A_{*}$ such that

$$
\{u\}_{,}^{\prime}=\{x\}^{\prime}=\{e(x)\}_{,}^{\prime} .
$$

Therefore, by Theorem 2.5, $u$ is equal to $e(x)$ and, by Theorem 3.2, it follows that $u$ is $\sigma$-finite.

TheOrem 3.4. Let $A$ be a $\mathrm{JBW}^{*}$-triple. Let $\mathscr{U}(A)$ be the partially ordered set of tripotents and let $\mathscr{U}_{\sigma}(A)$ be the set of $\sigma$-finite tripotents in $A$. Then:

(i) Let $\left(u_{n}\right)$ be a sequence in $\mathscr{U}_{\sigma}(A)$. If the supremum $\bigvee_{n=1}^{\infty} u_{n}$ of this sequence exists then the element $\bigvee_{n=1}^{\infty} u_{n}$ lies in $\mathscr{U}_{\sigma}(A)$.

(ii) Every element $u$ in $\mathscr{U}(A)$ is the supremum of an orthogonal subset of $\mathscr{U}_{\sigma}(A)$.

Proof. (i) We may assume that the elements in the sequence are nonzero. By Theorem 1, there exists a sequence $\left(x_{n}\right)$ of elements of norm one in $A_{*}$ such that $u_{n}$ is equal to $e\left(x_{n}\right)$. Let

$$
y_{m}=\sum_{n=1}^{m} \frac{1}{2^{n}} x_{n} .
$$

Then $\left(y_{m}\right)$ is a Cauchy sequence in $A_{*}$ which converges to an element $x$ in $A_{*, 1}$.

Now suppose that the supremum $\bigvee_{n=1}^{\infty} u_{n}$ exists. Then, by Theorem 2.5, 


$$
\left\{\bigvee_{n=1}^{\infty} u_{n}\right\},^{\prime}=\bigcap_{n=1}^{\infty}\left\{u_{n}\right\}_{\prime^{\prime}}=\bigcap_{n=1}^{\infty}\left\{e\left(x_{n}\right)\right\}_{\prime^{\prime}}=\bigcap_{n=1}^{\infty}\left\{x_{n}\right\}^{\prime}=\{x\}^{\prime} .
$$

By Corollary 3.3 and Corollary 2.6, it follows that $\bigvee_{n=1}^{\infty} u_{n}$ is a $\sigma$-finite tripotent.

(ii) If $u$ is a non-zero tripotent then $\{u\}$, is a non-empty subset of $A_{*, 1}$. Let $M$ be a maximal orthogonal set of elements in $\left\{e(x): x \in\{u\}_{1}\right\}$. By Theorem 2.3, it follows that $\bigvee_{v \in M} v$ exists. Moreover, $\bigvee_{v \in M} v \leq u$. By Theorem 2.1 (iv), there exists a tripotent $w$ such that

$$
\bigvee_{v \in M} v \perp w, \quad u=w \vee \bigvee_{v \in M} v .
$$

If $w$ is a non-zero tripotent then there exists an element $y$ in $A_{*, 1}$ which lies in $\{w\}$, and therefore lies in $\{u\}$, Since $e(y) \leq w$, by Theorem 2.1 (iv), it follows that $e(y) \perp M$ thereby violating the maximality of $M$.

Corollary 3.5. Let $A$ be a $\mathrm{JBW}^{*}$-triple and let $\mathscr{U}_{\sigma}(A)$ be the collection of its $\sigma$-finite tripotents. Then

$$
A=\varlimsup_{\operatorname{lin} \mathscr{U}_{\sigma}(A)}^{w^{*}} .
$$

Proof. This follows from Theorem 3.4 and Theorem 2.3.

Lemma 3.6. Let $A$ be a $\mathrm{JBW}^{*}$-triple.

(i) Let $B$ be a weak ${ }^{*}$-closed subtriple in A. Then

$$
\mathscr{U}_{\sigma}(A) \cap B \subseteq \mathscr{U}_{\sigma}(B) .
$$

(ii) Let $J$ be a weak ${ }^{*}$-closed inner ideal in $A$. Then

$$
\mathscr{U}_{\sigma}(A) \cap J=\mathscr{U}_{\sigma}(J) .
$$

Proof. The proof of (i) is immediate. To prove (ii) let $u$ be an element in $\mathscr{U}_{\sigma}(J)$ and let $M$ be an orthogonal subset of $\mathscr{U}(A)$ such that $M \leq u$. Then, by [7], Lemma 2.1, $M$ is a subset of $J$ and, thus, $M$ is at most countable. Therefore the tripotent $u$ lies in $\mathscr{U}_{\sigma}(A) \cap J$.

Corollary 3.7. Let $A$ be a $\mathrm{JBW}^{*}$-triple.

(i) Let $B_{1}, B_{2}$ be weak ${ }^{*}$-closed subtriples in $A$ such that $\mathscr{U}_{\sigma}\left(B_{1}\right)$ is contained in $\mathscr{U}_{\sigma}\left(B_{2}\right)$. Then $B_{1}$ is contained in $B_{2}$.

(ii) Let $J_{1}, J_{2}$ be weak ${ }^{*}$-closed inner ideals in $A$. Then $\mathscr{U}_{\sigma}\left(J_{1}\right)$ is contained in $\mathscr{U}_{\sigma}\left(J_{2}\right)$ if and only if $J_{1}$ is contained in $J_{2}$.

PROOF. These follow from Corollary 3.5 and Lemma 3.6.

THEOREM 3.8. Let $A$ be a $\mathrm{JBW}^{*}$-triple and let $B$ be a weak*-closed subtriple 
of $A$. Then $B$ is an inner ideal if and only if, for every element $u$ in $\mathscr{U}_{\sigma}(B)$, the weak ${ }^{*}$-closed subtriple $A_{2}(u)$ is contained in $B$.

Proof. Let $u$ be any tripotent in $B$. By Theorem 3.4 (ii) and Theorem 2.3, there exists an increasing net $\left(u_{\gamma}\right)_{\gamma \in \Gamma}$ in $\mathscr{U}_{\sigma}(B)$ which converges to $u$ in the weak*-topology. Since, for all elements $\gamma$ in $\Gamma, u_{\gamma} \leq u$ it follows that $\left(u_{\gamma}\right)_{\gamma \in \Gamma}$ is an increasing net of self-adjoint idempotents in the $\mathrm{JBW}^{*}$-algebra $A_{2}(u)$. By [22], 4.1.3, it follows that this net converges to $u$ in the strong topology of the $\mathrm{JBW}^{*}$-algebra $A_{2}(u)$. The Jordan multiplication in $A_{2}(u)$ being jointly strongly continuous on norm-bounded sets, by [22], Lemma 4.19, for every element $a$ in $A_{2}(u)$, the net $\left(\left\{u_{\gamma} a u_{\gamma}\right\}\right)_{\gamma \in \Gamma}$ converges in the strong topology of $A_{2}(u)$ and, hence, in the weak*-topology of $A$ to the element $\{u a u\}$. Since $A_{2}\left(u_{\gamma}\right)$ is an inner ideal in $A$, by the hypothesis, it follows that the net $\left(\left\{u_{\gamma} a u_{\gamma}\right\}\right)_{\gamma \in \Gamma}$ and, therefore, the element $\{u a u\}$ lies in $B$. Finally, the element $a$ which is equal to $\{u\{u a u\} u\}$ is contained in $B$.

It has been shown that, for every tripotent $u$ in $B, A_{2}(u)$ is contained in $B$. By [7], Lemma $2.1, B$ is an inner ideal in $A$. The converse is an immediate consequence of the same lemma.

\section{The Main Results.}

Let $A$ be a $\mathrm{JBW}^{*}$-triple with predual Banach space $A_{*}$. Let $x$ be an element in $A_{*}$ of norm one and let $e(x)$ be its support. By [2], Proposition 1.2, the mapping $\phi_{x}: A \times A \rightarrow \mathrm{C}$ defined, for elements $a, b$ in $A$, by

$$
\phi_{x}(a, b)=x(\{a b e(x)\})
$$

is a positive sesquilinear form on $A$ satisfying the Cauchy-Schwarz inequality

$$
\left|\phi_{x}(a, b)\right|^{2} \leq \phi_{x}(a, a) \phi_{x}(b, b) .
$$

The following result can be extracted from [2].

Lemma 4.1. Let $A$ be a $\mathrm{JBW}^{*}$-triple and let $x$ be an element in $A_{*}$ of norm one. Let $\phi_{x}$ be the sesquilinear form on $A$ defined above. Then, for elements a in $A$,

$$
\phi_{x}(a, a)=0
$$

if and only if a lies in $A_{0}(e(x))$.

Proof. Let $a$ be an element in $A$ such that $\phi_{x}(a, a)$ is equal to 0 . For $i$ equal to 0,1 or 2 , denote the element $P_{i}(e(x))(a)$ by $a_{i}$. By the hermiticity of $\phi_{x}$ and the Peirce rules, 


$$
\begin{aligned}
\phi_{x}(a, a)= & \phi_{x}\left(a_{2}+a_{1}, a_{2}+a_{1}\right)+2 \operatorname{Re}\left(x\left(\left\{a_{2}+a_{1} a_{0} e(x)\right\}\right)\right) \\
& +x\left(\left\{a_{0} a_{0} e(x)\right\}\right) \\
= & \phi_{x}\left(a_{2}, a_{2}\right)+2 \operatorname{Re}\left(x\left(\left\{a_{2} a_{1} e(x)\right\}\right)\right)+\phi_{x}\left(a_{1}, a_{1}\right) .
\end{aligned}
$$

Therefore

$$
x\left(\left\{a_{2} a_{2} e(x)\right\}\right)=x\left(\left\{a_{1} a_{1} e(x)\right\}\right)=0 .
$$

The element $a_{2} \circ a_{2}{ }^{\dagger}$, which is equal to $\left\{a_{2} a_{2} e(x)\right\}$, lies in the positive cone of the $\mathrm{JBW}^{*}$-algebra $A_{2}(e(x))$. Since the restriction of $x$ to $A_{2}(e(x))$ yields a faithful state on $A_{2}(e(x))$ it follows that $a_{2}$ is equal to 0 . On the other hand, by [19], Lemma 1.5, $\left\{a_{1} a_{1} e(x)\right\}$ is also a positive element in $A_{2}(e(x))$ and therefore equal to zero. It follows from the same lemma that $a_{1}$ is zero. The converse is immediate.

We shall need the following result.

Lemma 4.2. Let $A$ be a $\mathrm{JBW}^{*}$-triple and let $u$ be a $\sigma$-finite tripotent in $A$. Let $v$ be a tripotent in $A$ such that

$$
A_{2}(v)_{\mathrm{sa}} \cap A_{0}(u)=\{0\} .
$$

Then $v$ is $\sigma$-finite.

Proof. Let $u$ be a non-zero $\sigma$-finite tripotent. Then, by Theorem 3.2, there exists an element $x$ of norm one in $A_{*}$ such that $e(x)$ is equal to $u$.

Now let $v$ be a tripotent satisfying the condition above. Let $M$ be a nonempty orthogonal subset of non-zero tripotents in $A$ majorized by $v$. Then $M$ is contained in $A_{2}(v)_{\text {sa }}$. Let $N$ be a non-empty finite subset of $M$. Then

$$
0 \leq \sum_{w \in N} \phi_{x}(w, w)=\phi_{x}\left(\sum_{w \in N} w, \sum_{w^{\prime} \in N} w^{\prime}\right) \leq 1
$$

Therefore the increasing real net $\left(\sum_{w \in N} \phi_{x}(w, w)\right)_{N \in M^{f}}$ is bounded and, hence, converges in $\mathrm{R}$. It follows that there exists a subset $L$ of $M$ which is at most countable such that, for all elements $w$ in $M \backslash L, \phi_{x}(w, w)$ is equal to zero. By Lemma 2 and the hypothesis, we conclude that $M \backslash L$ is empty.

Corollary 4.3. Let $A$ be a $\mathrm{JBW}^{*}$-triple and let $u$ be $a$-finite tripotent in $A$. Let $J$ be a weak* ${ }^{*}$-closed inner ideal in $A$ such that every non-zero tripotent in $J$ is not orthogonal to $u$. Then $J$ is $\sigma$-finite.

Proof. Let $v$ be a tripotent in $J$. By [7], Lemma 2.1, $A_{2}(v)$ is a subset of $J$. By hypothesis, the weak*-closed subtriple $A_{2}(v) \cap A_{0}(u)$ contains no nonzero tripotent and therefore coincides with $\{0\}$. By Lemma 4.2, it follows that $v$ is a $\sigma$-finite tripotent. 
The following result gives a characterization of $\sigma$-finite $\mathrm{JBW}^{*}$-triples in terms of the geometry of the unit ball and in terms of the partially ordered set of the tripotents.

Theorem 4.4. Let $A$ be a $\mathrm{JBW}^{*}$-triple with unit ball $A_{1}$. Then the following conditions are equivalent.

(i) Every weak ${ }^{*}$-closed face of $A_{1}$ is weak ${ }^{*}$-exposed.

(ii) Every extreme point of $A_{1}$ is weak ${ }^{*}$-exposed.

(iii) There exists a weak ${ }^{*}$-exposed point in $A_{1}$.

(iv) Every weak*-closed face of $A_{1}$ is norm-exposed.

(v) Every extreme point of $A_{1}$ is norm-exposed.

(vi) There exists a norm-exposed point in $A_{1}$.

(vii) Every maximal tripotent in $A$ is $\sigma$-finite.

(viii) There exists a $\sigma$-finite maximal tripotent in $A$.

(ix) The $\mathrm{JBW}^{*}$-triple $A$ is $\sigma$-finite.

Proof. The eight implications (i) $\Rightarrow$ (ii), (ii) $\Rightarrow$ (iii), (iii) $\Rightarrow$ (vi), (i) $\Rightarrow$ (iv), (iv) $\Rightarrow$ (v), (v) $\Rightarrow$ (vi), (ix) $\Rightarrow$ (vii) and (vii) $\Rightarrow$ (viii) are straightforward.

(vi) $\Rightarrow$ (viii): We may assume that $A$ is different from $\{0\}$. Suppose that $u$ is a norm-exposed point in $A_{1}$. Then $u$ is an extreme point of the unit ball $A_{1}$ and therefore, by Theorem 2.4, a maximal tripotent in $A$. Moreover, there exists an element $x$ of norm one in the Banach space dual $A^{*}$ of $A$ such that $\{x\}$, coincides with $\{u\}$. A straightforward modification of [10], Lemma 2.3, shows that the restriction of $x$ to $A_{2}(u)$ is a state on the $\mathrm{JBW}^{*}$-algebra $A_{2}(u)$. Let $v$ be a tripotent in $A$ such that $v \leq u$ and suppose that $x(v)$ is equal to zero. Then the tripotent $u-v$ lies in $\{x\}$, and consequently $v$ is equal to 0 .

Therefore, for all tripotents $v$ with $0<v \leq u, x(v)>0$. Now let $M$ be a non-empty orthogonal subset of non-zero tripotents for which $u$ is an upper bound. Let $N$ be a non-empty finite subset of $M$. Then

$$
0 \leq \sum_{w \in N} x(w)=x\left(\sum_{w \in N} w\right) \leq 1 .
$$

Hence the increasing real net $\left(\sum_{w \in N} x(w)\right)_{N \in M^{f}}$ is bounded and therefore converges. It follows that $M$ is at most countable. Consequently, the maximal tripotent $u$ is $\sigma$-finite.

(viii) $\Rightarrow$ (ix): Let $u$ be a $\sigma$-finite maximal tripotent. It follows that $A_{0}(u)$ is equal to $\{0\}$. Therefore, by Lemma 4.2, every tripotent in $A$ is $\sigma$-finite.

(ix) $\Rightarrow$ (i): By Theorem 2.5, every non-empty weak*-closed face of $A_{1}$ contains a tripotent of $A$. By Corollary 3.3, every weak*-closed face of $A_{1}$ is weak*-exposed. 


\section{Applications and Examples.}

It is clear that every finite-dimensional $\mathrm{JBW}^{*}$-triple is $\sigma$-finite. The first example of a $\mathrm{JBW}^{*}$-triple to be considered, which is not necessarily finite-dimensional, is that of a $\mathrm{W}^{*}$-algebra $A$, for the properties of which the reader is referred to [27] and [28]. The triple product of elements $a, b$ and $c$ in the $\mathrm{W}^{*}$-algebra $A$ is given by

$$
\{a b c\}=\frac{1}{2}\left(a b^{*} c+c b^{*} a\right) .
$$

For the proof that, with this triple product, the $\mathrm{W}^{*}$-algebra $A$ does indeed form a $\mathrm{JBW}^{*}$-triple the reader is referred to [29]. The set of partial isometries in $A$ coincides with the set $\mathscr{U}(A)$ of tripotents in $A$. For $u$ and $v$ in $\mathscr{U}(A)$, $u \leq v$ if and only if $u v^{*} u=u$ and $u \perp v$ if and only if $u v^{*}=v^{*} u=0$. Let $\mathscr{P}(A)$ denote the set of self-adjoint idempotents, the projections, in $A$. By [10], Lemma 2.4, the set $\mathscr{P}(A)$ coincides with the order interval $[0,1]$ of $\mathscr{U}(A)$, where 1 is the multiplicative unit in $A$. Observe that, for elements $p$ and $q$ in $\mathscr{P}(A), p \leq q$ if and only if $p q=p$ and $p \perp q$ if and only if $p q=0$. By Corollary 2.2, $\mathscr{P}(A)$ is a complete lattice. Moreover, for elements $u$ and $v$ in $\mathscr{U}(A), u \perp v$ if and only if $u u^{*} \perp v v^{*}$ and $u^{*} u \perp v^{*} v$. For details of these results the reader is referred to [18].

A $\mathrm{W}^{*}$-algebra $A$ is said to be $\sigma$-finite if every orthogonal subset of $\mathscr{P}(A)$ is at most countable [28]. The results of $\S 4$ lead to the following theorem.

Theorem 5.1. Let $A$ be a $W^{*}$-algebra with unit ball $A_{1}$. Then the following conditions are equivalent.

(i) Every weak ${ }^{*}$-closed face of $A_{1}$ is weak wexposed. $^{*}$

(ii) Every extreme point of $A_{1}$ is weak ${ }^{*}$-exposed.

(iii) There exists a weak ${ }^{*}$-exposed point in $A_{1}$.

(iv) Every weak*-closed face of $A_{1}$ is norm-exposed.

(v) Every extreme point of $A_{1}$ is norm-exposed.

(vi) There exists a norm-exposed point in $A_{1}$.

(vii) Every maximal partial isometry in $A$ is $\sigma$-finite.

(viii) There exists a $\sigma$-finite maximal partial isometry in $A$.

(ix) Every partial isometry in $A$ is $\sigma$-finite.

(x) The unit 1 in $A$ is $\sigma$-finite.

(xi) $A$ is $\sigma$-finite.

(xii) A admits a faithful normal state.

(xiii) A admits a faithful state.

Proof. The equivalence of the conditions (i) - (ix) follows from Theorem 4.4. The implications (ix) $\Rightarrow(\mathrm{x})$ and $(\mathrm{x}) \Rightarrow$ (xi) are straightforward.

(xi) $\Rightarrow$ (xii): This follows from [8], Theorem 4.6. 
(xii) $\Rightarrow$ (xiii): This is obvious.

(xiii) $\Rightarrow(\mathrm{x})$ : This follows by standard arguments and the fact that $p \leq 1$ implies that $p$ lies in $\mathscr{P}(A)$.

$(\mathrm{x}) \Rightarrow$ (viii): Notice that, by direct computation, $A_{0}(1)$ coincides with $\{0\}$. Therefore 1 is a maximal element in $\mathscr{U}(A)$.

The following result, presented in [21], appears as a corollary of Theorem 5.1 .

COROLlary 5.2. The following conditions on the $W^{*}$-algebra $B(H)$ of bounded operators on the Hilbert space $H$ are equivalent.

(i) $H$ is separable.

(ii) The unit ball in $B(H)$ contains a norm-exposed point.

(iii) Every extreme point of the unit ball in $B(H)$ is norm-exposed.

The results of $\$ 4$ can be applied to various sub-JBW*-triples of a $\mathrm{W}^{*}$-algebra $A$. Let $\alpha$ be a *anti-automorphism of $A$ of order two and let $\alpha^{*}$ denote the adjoint mapping on the dual $A^{*}$ of $A$. Then $\alpha^{*}$ is a positive linear isometry from $A^{*}$ onto itself and therefore restricts to an affine automorphism of the state space of $A$. Since the predual $A_{*}$ of $A$ is unique, $\alpha^{*}$ restricts to a positive linear isometry from $A_{*}$ onto itself which again restricts to an affine automorphism of the normal state space of $A$. Let $H(A, \alpha)$ denote the subset of $A$ consisting of $\alpha$-invariant elements of $A$. Then $H(A, \alpha)$ is a sub-JBW*triple of $A$ and is also a $\mathrm{JBW}^{*}$-algebra with multiplication defined, for elements $a$ and $b$ in $H(A, \alpha)$, by

$$
a \circ b=\frac{1}{2}(a b+b a) .
$$

When restricted to $H(A, \alpha)$ the triple product of elements $a, b$ and $c$ in $H(A, \alpha)$ coincides with the Jordan triple product

$$
\{a b c\}=a \circ\left(b^{*} \circ c\right)+\left(a \circ b^{*}\right) \circ c-b^{*} \circ(a \circ c) .
$$

It is clear that the dual $H(A, \alpha)^{*}$ of $H(A, \alpha)$ can be identified with the closed subspace $H\left(A^{*}, \alpha^{*}\right)$ of $A^{*}$ consisting of $\alpha^{*}$-invariant elements and the predual $H(A, \alpha)_{*}$ of $H(A, \alpha)$ can be identified with the closed subspace $H\left(A_{*}, \alpha^{*}\right)$ of $A_{*}$ consisting of $\alpha^{*}$-invariant elements.

Recall that the $\mathrm{JBW}^{*}$-algebra $H(A, \alpha)$ is said to be $\sigma$-finite if every orthogonal subset of the complete lattice $\mathscr{P}(H(A, \alpha))$ of self-adjoint idempotents in $H(A, \alpha)$ is at most countable. An element $e$ in $\mathscr{P}(H(A, \alpha))$ is said to be $\sigma$ finite if the $\mathrm{JBW}^{*}$-algebra $H\left(e A e,\left.\alpha\right|_{e A e}\right)$ is $\sigma$-finite. The results of $\S 4$ lead to the following theorem.

Theorem 5.3. Let $A$ be a $W^{*}$-algebra, let $\alpha$ be $a^{*}$-anti-automorphism of $A$ of order two, let $H(A, \alpha)$ be the $\mathrm{JBW}^{*}$-algebra and sub-JBW*-triple of $A$ con- 
sisting of $\alpha$-invariant elements of $A$ and let $H(A, \alpha)_{1}$ be the unit ball in $H(A, \alpha)$. Then the following conditions are equivalent.

(i) Every weak ${ }^{*}$-closed face of $H(A, \alpha){ }_{1}$ is weak ${ }^{*}$-exposed.

(ii) Every extreme point of $H(A, \alpha)_{1}$ is weak ${ }^{*}$-exposed.

(iii) There exists a weak*-exposed point in $H(A, \alpha)_{1}$.

(iv) Every weak ${ }^{*}$-closed face of $H(A, \alpha)_{1}$ is norm-exposed.

(v) Every extreme point in $H(A, \alpha)_{1}$ is norm-exposed.

(vi) There exists a norm-exposed point in $H(A, \alpha)_{1}$.

(vii) Every maximal element in $\mathscr{U}(H(A, \alpha))$ is $\sigma$-finite.

(viii) There exists a $\sigma$-finite maximal element in $\mathscr{U}(H(A, \alpha))$.

(ix) Every element in $\mathscr{U}(H(A, \alpha))$ is $\sigma$-finite.

(x) The unit 1 in $\mathscr{U}(H(A, \alpha))$ is $\sigma$-finite.

(xi) $H(A, \alpha)$ is $\sigma$-finite.

(xii) $A$ admits an $\alpha^{*}$-invariant normal state, faithful on $H(A, \alpha)$.

(xiii) $A$ admits an $\alpha^{*}$-invariant state, faithful on $H(A, \alpha)$.

Proof. The equivalence of the conditions (i)-(ix) follows from Theorem 4.4. The implications (ix) $\Rightarrow(\mathrm{x})$ and $(\mathrm{x}) \Rightarrow$ (xi) are straightforward, (xi) $\Rightarrow$ (xii) follows from [8], Theorem 4.6 and (xii) $\Rightarrow$ (xiii) is immediate. The implication (xiii) $\Rightarrow(\mathrm{x})$ follows from the fact that, if $p$ is an element of $\mathscr{U}(H(A, \alpha))$ such that $p \leq 1$ then $p$ lies in $\mathscr{P}(H(A, \alpha))$. Notice that, by direct computation, $A_{0}(1)$ coincides with $\{0\}$. As a result, 1 is a maximal element in $\mathscr{U}(H(A, \alpha))$, thereby proving the final implication (x) $\Rightarrow$ (viii).

Recall that a $\mathrm{W}^{*}$-algebra the centre of which consists of complex multiples of its identity 1 is said to be a $W^{*}$-factor. Let $e$ and $f$ be elements of the complete lattice $\mathscr{P}(A)$ of projections in the $\mathrm{W}^{*}$-factor $A$. Then $e A f$ is a sub$\mathrm{JBW}^{*}$-triple of $A$ and is in fact an inner ideal in $A$. For a discussion of the properties of such inner ideals the reader is referred to [11].

TheOREM 5.4. Let $A$ be $a W^{*}$-factor and let $e$ and $f$ be projections in $A$. Then the $\mathrm{JBW}^{*}$-triple eAf is $\sigma$-finite if and only if at least one of the projections $e, f$ is $\sigma$-finite.

Proof. Suppose that $e$ is $\sigma$-finite and let $M$ be an orthogonal set of partial isometries in $e A f$. Then the set $\left\{u u^{*}: u \in M\right\}$ is an orthogonal set of projections in $A$ such that, for all $u$ in $M, u u^{*} \leq e$. Since $e$ is $\sigma$-finite the set is at most countable and, therefore, $M$ is also at most countable. A similar proof shows that if $f$ is $\sigma$-finite then so also is $e A f$.

Suppose now that $e A f$ is $\sigma$-finite and let $M$ and $N$ be orthogonal sets of non-zero projections in $A$, such that, for all $p$ in $M, p \leq e$, and, for all $q$ in $N$, $q \leq f$. Then, for all $p$ in $M$ and $q$ in $N, p A q$ is a sub-JBW ${ }^{*}$-triple of $e A f$. Observe that, if $p A q=\{0\}$, then, by [28], Proposition 1.10.7, either $p$ or $q$ is 
zero, yielding a contradiction. Therefore, $p A q$ is non-zero and there exists a non-zero partial isometry $u_{p q}$ in $p A q$ such that

$$
u_{p q} u_{p q}{ }^{*} \leq p \leq e, \quad u_{p q}{ }^{*} u_{p q} \leq q \leq f .
$$

Observe that, for $p_{1}$ and $p_{2}$ in $M$ with $p_{1} \neq p_{2}$ and $q_{1}$ and $q_{2}$ in $N$ with $q_{1} \neq q_{2}$, since $p_{1} \perp p_{2}$ and $q_{1} \perp q_{2}$, the partial isometries $u_{p_{1} q_{1}}$ and $u_{p_{2} q_{2}}$ are orthogonal. Since $e A f$ is $\sigma$-finite it follows that at least one of the sets $M$ or $N$ is at most countable and, as a consequence, at least one of the projections $e$ or $f$ must be $\sigma$-finite.

The next example considered is that of a spin triple. Let $H$ be a complex Hilbert space and let $a \mapsto \bar{a}$ be a conjugation on $H$. For elements $a, b$ and $c$ in $H$, let

$$
\{a b c\}=\langle a, b\rangle c+\langle c, b\rangle a-\langle a, \bar{c}\rangle \bar{b},
$$

where $\langle a, b\rangle$ denotes the inner product in $H$ of the elements $a$ and $b$. Then, using the results of [26], $H$ is a $\mathrm{JBW}^{*}$-triple with respect to the norm, which is equivalent to the Hilbert space norm, given by

$$
\|a\|^{2}=\langle a, a\rangle+\left(\langle a, a\rangle^{2}-|\langle a, \bar{a}\rangle|^{2}\right)^{\frac{1}{2}} .
$$

This $\mathrm{JBW}^{*}$-triple is said to be a spin triple. Observe that the set of non-zero tripotents in $H$ consists of the elements $u$ in $H$ such that $\langle u, \bar{u}\rangle=0$ and $\langle u, u\rangle=\frac{1}{2}$ along with those elements $u$ in $H$ for which $\bar{u}=\lambda u$, where $\lambda$ is a complex number of unit modulus, and $\langle u, u\rangle=1$. The set of tripotents orthogonal to a tripotent $u$ for which $\langle u, u\rangle=\frac{1}{2}$ consists of tripotents of the form $\lambda \bar{u}$, where $\lambda$ is a complex number of unit modulus. There are no nonzero tripotents orthogonal to a tripotent $u$ for which $\langle u, u\rangle=1$. It follows that every orthogonal subset of non-zero elements of $H$ consists of at most two elements, thereby leading to the following result.

THEOREM 5.5. A spin triple $H$ is a $\sigma$-finite $\mathrm{JBW}^{*}$-triple.

A final example of a $\mathrm{JBW}^{*}$-triple is a complex Hilbert space $H$ endowed with the triple product defined, for elements $a, b$ and $c$ in $H$, by

$$
\{a b c\}=\frac{1}{2}(\langle a, b\rangle c+\langle c, b\rangle a) .
$$

The set of non-zero tripotents in $H$ consists of elements $u$ of $H$ such that $\langle u, u\rangle=1$. There are no non-zero tripotents orthogonal to such an element $u$. This leads to the following result.

THEOREM 5.6. Let $H$ be a complex Hilbert space endowed with the triple product defined above. Then $H$ is a $\sigma$-finite $\mathrm{JBW}^{*}$-triple. 


\section{REFERENCES}

1. T. J. Barton, T. Dang, G. Horn,Normal representations of Banach Jordan triple systems, Proc. Amer. Math. Soc. 102 (1987), 551-555.

2. T. Barton, Y. Friedman, Grothendieck's inequality for JB*-triples and applications, J. London Math. Soc. 36 (1987), 513-523.

3. T. J. Barton, R. M. Timoney, Weak ${ }^{*}$ continuity of Jordan triple products and its applications, Math. Scand. 59 (1986), 177-191.

4. M. Battaglia, Order theoretic type decomposition of $\mathrm{JBW}^{*}$-triples, Quart. J. Math. Oxford 42 (1991), 129-147.

5. S. Dineen, Complete holomorphic vector fields in the second dual of a Banach space, Math. Scand. 59, (1986), 131-142.

6. C. M. Edwards, On Jordan $W^{*}$-algebras, Bull. Sci. Math. 2e série 104, (1980), 393-403.

7. C. M. Edwards, K. McCrimmon, G.T. Rüttimann, The range of a structural projection, J. Funct. Anal. 129 (1996), 196-241.

8. C. M. Edwards, G. T. Rüttimann, On the facial structure of the unit balls in a GL-space and its dual, Math. Proc. Camb. Philos. Soc. 98 (1985), 305-322.

9. C. M. Edwards, G. T. Rüttimann, On the facial structure of the unit balls in a GM-space and its dual, Math. Z. 193, 597-611 (1986).

10. C. M. Edwards, G. T. Rüttimann, On the facial structure of the unit balls in a $\mathrm{JBW}^{*}$-triple and its predual, J. London Math. Soc. 38 (1986), 317-332.

11. C. M. Edwards, G. T. Rüttimann, Inner ideals in $W^{*}$-algebras. Mich. Math. J. 36 (1989), $147-159$.

12. C. M. Edwards, G. T. Rüttimann, A characterization of inner ideals in JB*-triples, Proc. Amer. Math. Soc. 116 (1992), 1049-1057.

13. C. M. Edwards, G. T. Rüttimann, Structural projections on JBW*-triples, J. London Math. Soc. 53 (1996), 354-368.

14. C. M. Edwards, G. T. Rüttimann, Compact tripotents in bi-dual JB*-triples, Math. Proc. Camb. Philos. Soc. 120 (1996), 155-173.

15. C. M. Edwards, G. T. Rüttimann, Smoothness properties of the unit ball in a JB*-triple, Bull. London Math. Soc. 28 (1996), 156-160.

16. C. M. Edwards, G. T. Rüttimann, Peirce inner ideals in Jordan*triples, Journal of Algebra 180 (1996), 41-66.

17. C. M. Edwards, G. T. Rüttimann and S. Vasilovsky, Inner ideals in exceptional JBW*-triples, Michigan Math. J. 40 (1993), 139-152.

18. C. M. Edwards, G. T. Rüttimann and S. Vasilovsky, Invariant inner ideals in $W^{*}$-algebras, Math. Nachr. 172 (1995), 95-108.

19. Y. Friedman, B. Russo, Structure of the predual of a JBW*-triple, J. Reine Angew. Math. 356 (1985), 67-89.

20. Y. Friedman, B. Russo, The Gelfand-Naimark theorem for JB*-triples, Duke Math. J. 53 (1986) 139-148.

21. R. Grzaslewicz, Exposed points of the unit ball of $\mathscr{L}(H)$, Math. Z. 193 (1986), 595-596.

22. H. Hanche-Olsen, E. Størmer, Jordan Operator Algebras, Pitman, London 1984.

23. G. Horn, Characterization of the predual and the ideal structure of a $\mathrm{JBW}^{*}$-triple, Math. Scand. 61 (1987), 117-133.

24. N. Jacobson, Structure and representation of Jordan algebras, (Amer. Math. Soc. Colloquium Publication 39). American Mathematical Society, Providence 1968.

25. O. Loos, Jordan pairs, Lecture Notes in Math. 460, 1975.

26. E. Neher, Jordan triple systems by the grid approach, Lecture Notes in Math. 1280, 1987.

27. G.K.Pedersen, $C^{*}$-algebras and their automorphism groups, London Mathematical Society Monographs 14, Academic Press, London ,1979. 
28. S. Sakai, $C^{*}$-algebras and $W^{*}$-algebras, Springer Berlin, Heidelberg, New York, 1971.

29. H. Upmeier, Symmetric Banach manifolds and Jordan $C^{*}$-algebras, North Holland, Amsterdam, New York, Oxford, 1985.

30. J. D. M. Wright, Jordan $C^{*}$-algebras, Michigan Math. J. 24 (1977), 291-302.

31. M. A. Youngson, A Vidav theorem for Banach Jordan algebras, Math. Proc. Camb. Philos. Soc. 84 (1978), 263-272.

THE QUEEN'S COLLEGE OXFORD

UNITED KINGDOM
UNIVERSITY OF BERNE BERNE

SWITZERLAND 